\title{
On Numerical Modeling of Chaotic Advection and Diffusion in Fractured- Porous Media
}

\author{
BORIS FAYBISHENKO AND JENS BIRKHOLZER
}

Lawrence Berkeley National Laboratory

Presenting Author: bafaybishenko@lbl.gov

Experimental and theoretical studies indicate that coupled chemical and physical nonlinear dynamical processes often exhibit features of chaotic mixing, including chaotic advection and chaotic diffusion, which significantly deviate from classical transport in porous media. Nonlinear dynamical processes are inherent in flow and transport through fractured-porous media, including sediments (granular media) and fractured rock. Chaotic mixing processes are generated due to the intrinsic topological complexity of the pore-scale and fracture network architecture. In this presentation, we will review factors and processes of flow and transport in fractured-porous media, based on field and laboratory tests, which could cause phenomena of chaotic mixing, advection and diffusion, and will demonstrate an application of numerical reduced order models for simulations of thermo-hydro-chemical transport in fractured porous media. Modeling was performed using two models: (a) Darcy-Brinkman (DB) system of equations, reduced to four ordinary differential equations, and (b) Fisher-Kolmogorov-Petrovsky-Piskunov (FKPP) partial differential equations, describing diffusionreaction processes, including a logistic function term added to the transport terms. The solutions of the system of DB equations, with different sets of input parameters, demonstrate evidence of transition from steady-state to periodic to deterministic chaotic behavior, and then to random phenomena. The Bayesian inference network analysis is then used to illustrate the conditional dependence, and therefore causation of outputs of the DB model predictions. The results of simulations can be used for developing hypotheses and directing further experimental and modeling research needed for the design of nuclear disposal facilities, as well to develop approaches for controlling hydrothermo-chemical processes at multiple scales. 